\title{
Educando en periodismo social de un modo innovador
}

\section{Teaching in social journalism in an innovative way}

RUBÉn Domínguez-DELGADO

http://orcid.org/0000-0001-9885-2831

Universidad de Sevilla

Departamento de Periodismo I

rdd@us.es

DOI: http://dx.doi.org/10.12795/9788447231003.017

Pp.: 351-376 


\section{Introducción y contexto}

En este capítulo se da a conocer la experiencia de diseño y aplicación de un Ciclo de Mejora en el Aula (CIMA) en el grupo 1 de la asignatura Periodismo Social y Educativo, impartida durante el primer cuatrimestre del año académico 2020/2021 y en el cuarto curso del Grado en Periodismo, en la Facultad de Comunicación de la Universidad de Sevilla.

Este grupo cuenta con 71 alumnos matriculados, los cuales, para el periodo de docencia en el que se desarrolla este CIMA (de 8 horas, distribuidas en 4 sesiones de 2 horas cada una), y debido a la pandemia de COVID-19, siguen desde casa la clase de forma virtual a través de la plataforma de enseñanza Blackboard de la Universidad de Sevilla, así como mediante una cámara y un micrófono instalados en el aula física del Centro en la que se encuentra el profesor.

Se trata de una asignatura de carácter práctico en la que tenemos una cierta experiencia docente, al haberla impartido ininterrumpidamente desde el curso académico 2013/2014 y haber sido el coordinador de sus distintos grupos de actividad desde el curso 2015/2016. Algunos de sus principales objetivos son: observar cuáles son las rutinas productivas que determinan la representación de las realidades sociales en los medios y analizar su tratamiento informativo, conocer la situación de vulnerabilidad que sufren determinados colectivos sociales en la defensa de sus derechos, adquirir la capacidad crítica necesaria para la reconceptualización de las ideas dominantes que a diario condicionan el discurso periodístico, así como conocer las estrategias discursivas y recursos narrativos que se utilizan en los medios para dar forma a parte de la problemática social.

Ciclos de Mejora en el Aula (2020). Experiencias de Innovación Docente de la US Esta obra se distribuye con la licencia Creative Commons 


\section{Diseño previo del CIMA}

Objetivo y modelo metodológico posible

Nos planteamos como principal objetivo en la aplicación de este CIMA de 4 clases y 8 horas totales, que se desarrolla en plena introducción al periodismo social, en el bloque II de la asignatura (tras la impartición de una primera parte dedicada al periodismo educativo, por parte de otros profesores), reducir el tiempo dedicado a la tradicional clase magistral teórica (en nuestro caso, una delimitación conceptual y distintas aproximaciones a la materia por parte de diversos autores), para sustituirla por clases mucho más dinámicas y participativas, y más orientadas a la reflexión, a la aplicación práctica y a la conexión con una realidad y actualidad reconocible por parte de los estudiantes, con un peso importante del análisis, la crítica, el debate, la ética y la justicia social.

Por ello, se ha optado por un modelo metodológico posible (Porlán y Alba-Fernández, 2020:144-146) de tipo activo, constructivista e investigativo, con problemas y análisis no cerrados a discutir, debatir y contrastar, sucesivas actividades de diversa índole y un importante cierre final que ayude a asentar ideas mejoradas fundamentales, además de a dar coherencia al conjunto de actividades y al resto de la asignatura. Así, la representación gráfica de nuestro modelo sería la siguiente:

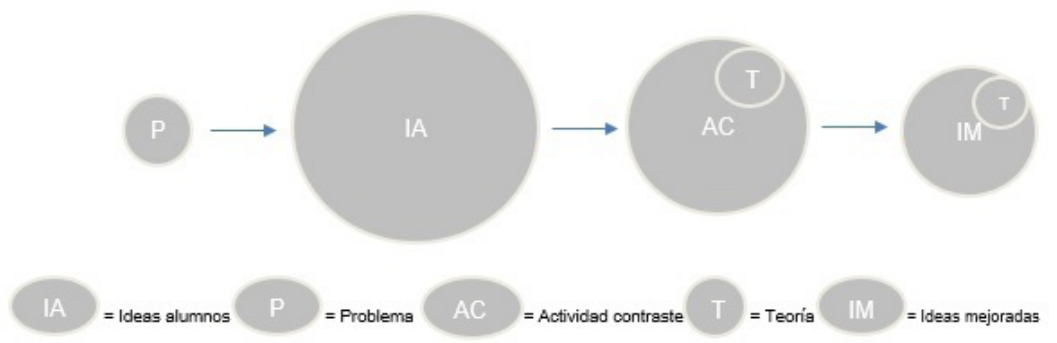

Figura 1. Modelo metodológico

Ciclos de Mejora en el Aula (2020). Experiencias de Innovación Docente de la US Esta obra se distribuye con la licencia Creative Commons 
Partimos de un problema inicial ("periodismo social: ¿para quién, para qué, por qué y cómo?"), que, aunque en realidad encierra cuatro subproblemas o interrogantes, nos ha parecido más conveniente agrupar, tanto en el modelo como en el mapa y en la secuencia de actividades, en uno solo, por estar los cuatro estrechamente entrelazados (haciéndose para cada uno continuas alusiones a los otros) y por disponer de una última actividad en nuestra secuencia que engloba a los cuatro por igual. Por tanto, partiendo de ese problema múltiple damos paso, para cada subproblema, bien a las ideas de los alumnos, con sometimiento siempre a un contraste posterior, o bien exclusivamente a una actividad de contraste. $Y$ en todas las actividades de contraste se insertarán siempre algunos aspectos teóricos importantes de la asignatura.

Para concluir, se lleva a cabo una actividad global de ideas mejoradas como cierre y síntesis de todo lo anterior, en la que también se incluyen elementos teóricos esenciales.

\section{Mapa de contenidos, problemas $y$ actividades del CIMA}

De la ordenación de los contenidos de la asignatura, estructurados en el programa y proyecto docente en 2 partes y 3 bloques, este CIMA se aplica en los contenidos de los epígrafes 1 y 5 del bloque II ("periodismo social") de la segunda parte de la asignatura, denominados "Introducción: definición y funciones del Periodismo Social" y "Tratamiento informativo a colectivos vulnerables: Inmigración, Infancia y Menores, Género, Etnias, Discapacidad, Tendencia sexual, etc.", respectivamente. En ellos, consideramos esenciales las referencias y aportaciones de autores como Cytrynblum, 2009; Raigón, 2007; Serrano, 2010; Gómez-Mompart et al, 2013; Rodríguez-Rey et al, 2015; López-Hernández y Domínguez-Delgado, 2012; Asensio-Pérez y Domínguez-Delgado, 2020; Castillo-Tamayo y Domínguez-Delgado, 
2020; Labio, 2007; Cruz Roja Española, 2015; Red Acoge, 2014; o FAPE, 2017, las cuales se han tenido en cuenta en la confección de nuestro mapa de contenidos. Así, el diseño del mapa de contenidos, problemas y actividades para las cuatro sesiones del CIMA sería el siguiente:

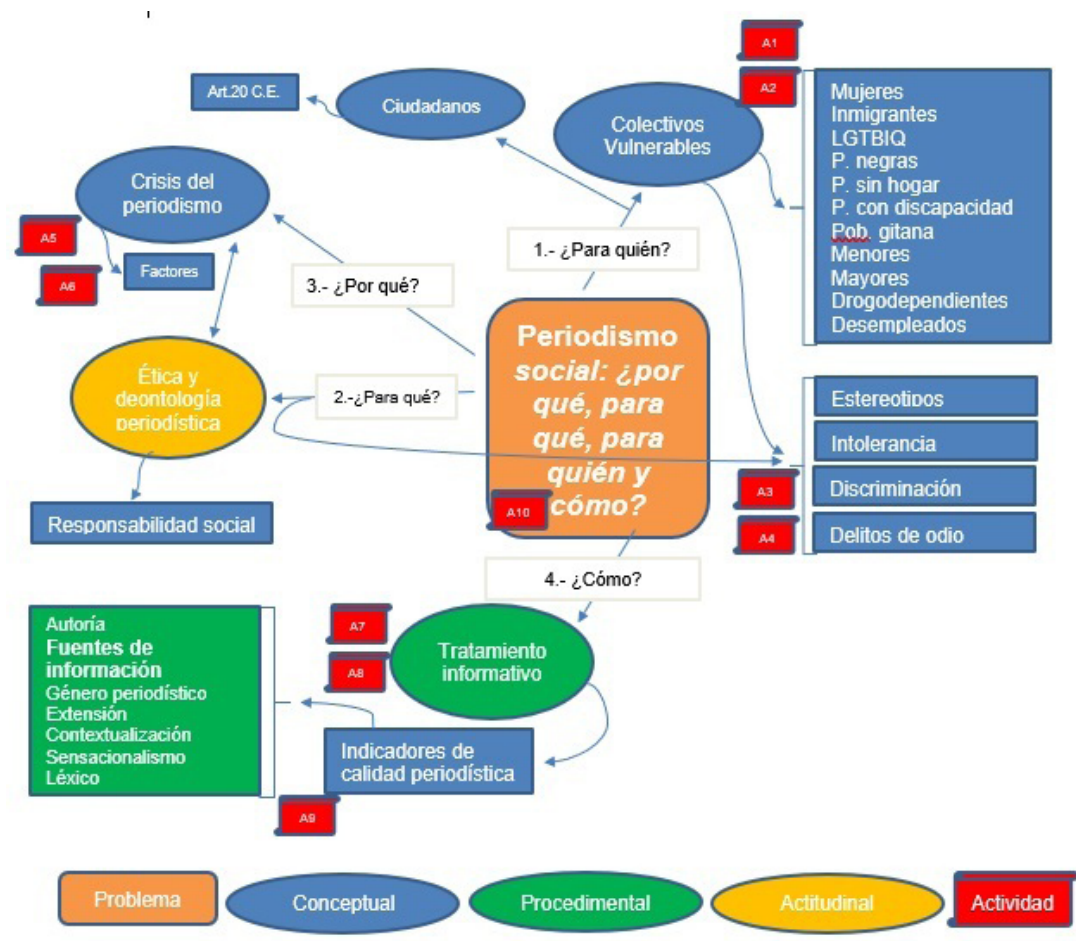

Figura 2. Mapa

Secuencia de actividades

ACTIVIDAD №1

- Nombre reconocible (fase del modelo metodológico): reflexión y discusión grupal (IA).

- Recurso Utilizado: salas virtuales y contenidos de Ev.us. es.

- Tiempo previsto: 20 minutos.

- Contenidos abordados: estereotipos sociales y colectivos vulnerables.

Ciclos de Mejora en el Aula (2020). Experiencias de Innovación Docente de la US (C) Esta obra se distribuye con la licencia Creative Commons Reconocimiento-NoComercial-SinObraDerivada Internacional (CC BY-NC-ND 4.0.) 
- Descripción de la actividad: se divide a los alumnos en grupos de 7 personas por orden alfabético de la lista de asistentes (10 grupos en total), creando una sala virtual para cada grupo, nombrando a todos sus integrantes moderadores de la sala (para que puedan compartir pantalla y documentos) y pidiendo a cada grupo que elija a un portavoz. Se asigna a cada equipo un grupo o colectivo social vulnerable distinto (mujeres, inmigrantes, población gitana, etc.) y se les pide, a modo de problema, para que reflexionen y discutan en el grupo, contestar a la pregunta siguiente: ¿qué estereotipos, prejuicios o ideas preconcebidas sobre el colectivo social creéis que existen en nuestro país, qué puede haber de verdad y de mentira en ellas y cómo puede afectar a la sociedad esas ideas o etiquetas?. El portavoz debe anotar por escrito los consensos alcanzados en el grupo y el profesor supervisará el trabajo de los distintos grupos y resolverá las dudas que se le planteen.

\section{ACTIVIDAD №2}

- Nombre reconocible (fase del modelo): presentación de conclusiones y discusión global (AC).

- Recurso Utilizado: salas virtuales y contenidos de Ev.us. es.

- Tiempo previsto: 40 minutos.

- Contenidos abordados: estereotipos sociales y colectivos vulnerables.

- Descripción de la actividad: en la sala virtual general de la clase, y activándose como moderador al portavoz de cada uno de los grupos establecidos para la actividad 1, se irá llamando por orden de número de grupo a cada portavoz, para que presente en 2 ó 3 minutos los consensos alcanzados en su grupo en relación con los estereotipos o ideas preconcebidas sobre el colectivo vulnerable asignado. Habrá retroalimentación del resto de la clase tras cada presentación y, en 
última instancia, del profesor, que irá orientando el debate con preguntas, provocando conflicto o contraste en las ideas de los alumnos, en los casos en los que sea necesario.

\section{ACTIVIDAD №3}

- Nombre reconocible (fase del modelo): análisis, reflexión y discusión grupal (AC).

- Recurso Utilizado: salas virtuales y contenidos de Ev.us. es, y web www.crimenesdeodio.info

- Tiempo previsto: 20 minutos.

- Contenidos abordados: delitos de odio, su conexión con el periodismo y los medios y función social del periodismo.

- Descripción de la actividad: a cada uno de los grupos formados para las actividades 1 y 2 , se les pide ahora, como actividad de contraste, que exploren y analicen el proyecto web www.crimenesdeodio.info, una herramienta creada por dos periodistas para la memoria de los crimenes de odio con resultado de muerte cometidos en el Estado español entre 1990 y 2020 (en la cual, por tipo de delito o por zona geográfica, se puede acceder a la ficha personal de cada víctima, con detalles sobre lo que les ocurrió, el tipo de crimen, cuál fue la respuesta judicial y una recopilación de noticias y vídeos a parecidos en los medios sobre el caso). Tras analizar la web y algunos de los trágicos casos, se deberá reflexionar y discutir sobre las posibles conexiones entre los crímenes de odio y el periodismo, así como la contribución que este último podría hacer para ayudar a evitarlos. Llegar a acuerdos y listarlos por escrito. El profesor supervisará el trabajo de los distintos grupos y resolverá las dudas que se le planteen.

ACTIVIDAD No4

- Nombre reconocible (fase del modelo): presentación de conclusiones, puesta en común y discusión global (IA). 
- Recurso Utilizado: salas virtuales de Ev.us.es.

- Tiempo previsto: 40 minutos.

- Contenidos abordados: delitos de odio, su conexión con el periodismo y los medios, y función social del periodismo.

- Descripción de la actividad: en la sala virtual general de la clase, se nombra moderadores a los portavoces de cada uno de los grupos establecidos para la actividad 1, para que puedan compartir pantalla y documentos con el resto de compañeros. A continuación, se va llamando a los portavoces de los grupos para que, interviniendo de forma voluntaria inicialmente, presenten oralmente de forma breve (en unos 5 minutos) los acuerdos de su grupo en la actividad anterior, a modo de puesta en común general y tras reelaborar los alumnos sus propias ideas a raíz de observado en la actividad 3. Al finalizar cada presentación, habrá retroalimentación del resto de alumnos y, en última instancia, del profesor. Se promoverá un clima participativo que invite al debate y discusión global sobre las posibles contribuciones sociales del periodismo para poner freno a los delitos de odio.

\section{ACTIVIDAD No5}

- Nombre reconocible (fase del modelo): resolución de problema, con reflexión y discusión grupal (AC).

- Recurso Utilizado: salas virtuales de Ev.us.es.

- Tiempo previsto: 60 minutos.

- Contenidos abordados: crisis del periodismo, ética y deontología profesional.

- Descripción de la actividad: con respecto a las actividades 3 y 4 de esta secuencia, en las que se reflexionaba sobre la importante labor social que puede llevar a cabo el periodismo, se plantea ahora una actividad de contraste (también lo será la actividad 6, ligada estrechamente a esta). Así, se divide a los alumnos en grupos de 7 personas, nombrando cada uno a un portavoz 
(se cambian los grupos con respecto a las cuatro actividades anteriores, asignando ahora números alternos por orden alfabético de lista y emplazándoles al mismo número de sala virtual grupal creadas para la ocasión). Se les da a los grupos por escrito, cada 8 minutos y en el chat de las distintas salas, un fragmento breve diferente (aunque el mismo para todos los grupos) de declaración, entrevista o cita de algún trabajo (escrito o audiovisual - en este último caso, se dejarán 20 minutos en vez de 8, por el visionado), pronunciado por un periodista y/o investigador de prestigio en el ámbito del periodismo, relacionados todos ellos con el estado actual de la profesión. Se pide a los alumnos que, para cada extracto, reflexionen y debatan acerca de los significados e implicaciones del mismo, identifiquen en él los distintos aspectos que se denuncien o planteen e indaguen en sus posibles efectos en la persona que ejerce el periodismo y en los ciudadanos y en las posibles soluciones para aspecto. Se pide nombrar un portavoz por equipo, que irá tomando notas con los acuerdos o consensos alcanzados en cada grupo, las cuales serán necesarias en la actividad posterior.

En realidad, aunque no demos de antemano esta información a los alumnos, en todos los casos estos profesionales denuncian la mala praxis de hoy, en lo relativo a aspectos distintos, en la profesión, y lo lejos que se encuentra hoy el periodismo de cumplir con su verdadera misión y con su compromiso social, encontrándose en una situación de crisis.

Los 6 fragmentos seleccionados y que se van dando a los grupos en un orden lógico, de cuestiones más generales a aspectos más concretos, en relación con los objetivos de la asignatura, son los siguientes:

1. Ryszard Kapuscinsky (2005): “Las malas personas no pueden ser buenos periodistas".

Ciclos de Mejora en el Aula (2020). Experiencias de Innovación Docente de la US Esta obra se distribuye con la licencia Creative Commons 
2. Kapuscinsky (1999): “Desde que está considerada como una mercancía, la información ha dejado de verse sometida a los criterios tradicionales de la verificación, la autenticidad o el error. Ahora se rige por las leyes del mercado".

3. José María García (2017): "En el periodismo, o besas la mano o te vas debajo del puente".

4. Video (de 11'): "Tracor TV: El reportaje de investigación en TV: la necesidad de investigar. Parte I". Fragmento de entrevista de José Antonio Puente a los periodistas de investigación Javier Chicote y Melchor Miralles (de 2011) sobre la necesidad de investigar por parte del periodista y el rol que este debe asumir en la sociedad como vigilante del poder: https:// www.youtube.com/watch?v=a19lgOPlEDk

5. Chicote (2006): “En el periodismo rutinario, las fuentes de información utilizadas, verdadero test de comprobación de la calidad de un trabajo periodístico, son en su mayoría oficiales y no van más allá de los puntos de vista institucionales, fiel reflejo de las palabras pronunciadas por la fuente sin ser sometidas por el periodista a un análisis, sino que se eligen en función de los intereses del medio de comunicación".

6. Secanella (1986): "La corrupción periodística empieza cuando solo se trabaja con una fuente, al servicio de ella".

El profesor supervisará el trabajo de los distintos grupos y resolverá las dudas que se le planteen, aunque evitará dar respuestas haciendo preferiblemente uso de preguntas.

ACTIVIDAD No6

- Nombre reconocible (fase del modelo): presentación de conclusiones, puesta en común y discusión global $(\mathrm{AC})$. 
- Recurso Utilizado: sala virtual de EV.us.es.

- Tiempo previsto: 60 minutos.

- Contenidos abordados: crisis del periodismo, ética y deontología profesional.

- Descripción de la actividad: en la sala virtual general de la clase, y a modo de lluvia de ideas, se irán proponiendo, desde los diferentes grupos, distintas ideas por parte de sus portavoces - tanto significados e implicaciones que subyacen en cada fragmento como posibles efectos en los periodistas y los ciudadanos y posibles soluciones de futuro que se planteen -, en 3-5 minutos, para cada uno de los extractos (podrán intervenir alternativamente para cada extracto de forma voluntaria o, en caso de no hacerlo ninguno, se irá llamando por orden de número a sus portavoces), con retroalimentación del resto de la clase tras su presentación y, en última instancia, del profesor, que irá orientado el debate con preguntas en los casos en los que sea necesario o este se esté alejando de las cuestiones de fondo a debatir que subyacen en cada extracto. En el fondo, con esta actividad de contraste se estaría analizando y reflexionando sobre la mala praxis (vulneración de los códigos éticos y deontológicos) en el periodismo actual y los distintos factores que lo han llevado a una situación de crisis y pérdida de credibilidad, así como tratando de entender la necesidad, en esa situación, de promover un periodismo social, en la línea de autores como Cytrynblum (2009), una de las principales referencias en la asignatura.

\section{ACTIVIDAD №7}

- Nombre reconocible (fase del modelo): búsqueda de información (AC).

- Recurso Utilizado: sala virtual de EV.us.es e Internet (Google, Mynews, videoteca de TVE a la carta, archivo de RNE y/o otros archivos informativos similares).

- Tiempo previsto: 30 minutos.

- Contenidos abordados: tratamiento informativo a colectivos vulnerables. 
- Descripción de la actividad: en grupos de 7 (de nuevo por orden alfabético esta vez, como en las primeras cuatro actividades), búsqueda de 4 informaciones periodísticas (en formato prensa, radio y/o televisión) protagonizadas por el colectivo vulnerable con el que trabajaron en la actividad 1, dos de ellas con un buen tratamiento informativo al colectivo, a juicio de los alumnos, y otras dos con uno malo. Se les insiste en que traten de seleccionar los más claros ejemplos posibles de buen y mal tratamiento informativo a sus colectivos. El profesor supervisará el trabajo de los distintos grupos y resolverá las dudas que se le planteen, revisando la selección de cada grupo al final.

\section{ACTIVIDAD N요}

- Nombre reconocible (fase del modelo): análisis de textos periodísticos (IA).

- Recurso Utilizado: sala virtual de Ev.us.es.

- Tiempo previsto: 60 minutos.

- Contenidos abordados: tratamiento informativo a colectivos vulnerables e indicadores de calidad periodística.

- Descripción de la actividad: se pide a los grupos de la actividad 7 que, dividiéndose en dos subgrupos y trabajando cada uno con un ejemplo de buen y de mal tratamiento periodístico, analicen críticamente y de forma comparativa los textos periodísticos seleccionados en la actividad anterior, prestando especial atención a los siguientes indicadores de calidad periodística o elementos periodísticos de interés (que se les da a través del chat por escrito): autoría, fuentes de información, extensión, género informativo, sensacionalismo, contextualización, fotografía que acompaña, terminología empleada y presencia o ausencia de estereotipos, prejuicios y/o generalizaciones. Al final, realizarán una puesta en común de los dos subgrupos para llegar a un consenso general dentro del grupo sobre el tratamiento dado al colectivo vulnerable. El profesor supervisará el trabajo de los distintos grupos y resolverá las dudas que se le planteen. 
- Nombre reconocible (fase del modelo): presentación de resultados de análisis de textos (AC).

- Recurso Utilizado: sala virtual de Ev.us.es.

- Tiempo previsto: 90 minutos.

- Contenidos abordados: tratamiento informativo a colectivos vulnerables e indicadores de calidad periodística.

- Descripción de la actividad: en la sala virtual general de la clase, el portavoz de cada grupo de la actividad 8 expone en 6-8 minutos las conclusiones a las que han llegado en su grupo en relación con el tratamiento informativo dado al colectivo vulnerable asignado en los textos periodísticos seleccionados, a la vez que comparte pantalla y muestra los aspectos o elementos de los textos que les han llevado a pensar que se ha dado un tratamiento adecuado o inadecuado. Tras cada presentación, habrá retroalimentación del resto de la clase, impulsada por el profesor, que irá orientando el debate con preguntas, provocando conflicto o contraste en las ideas de los alumnos, en los casos en los que sea necesario, a la vez que irá añadiendo elementos teóricos en relación con los análisis realizados por los alumnos.

\section{ACTIVIDAD №10}

- Nombre reconocible (fase del modelo): debate de puesta en común global (IM).

- Recurso Utilizado: sala virtual de Ev.us.es.

- Tiempo previsto: 60 minutos.

- Contenidos abordados: estereotipos sociales, delitos de odio, tratamiento informativo a colectivos vulnerables, indicadores de calidad periodística, crisis del periodismo y ética y deontología profesional.

- Descripción de la actividad: en la sala virtual general del curso, sobre una pizarra en blanco, el profesor va anotando, a modo de lluvia de ideas y de recordatorio, cierre y conclusión, las ideas fundamentales extraídas de la realización de las actividades de la 1 a la 9 de esta

Ciclos de Mejora en el Aula (2020). Experiencias de Innovación Docente de la US Esta obra se distribuye con la licencia Creative Commons 
secuencia, contrastando y conectando todas ellas e incluyendo algunas nociones teóricas fundamentales de la asignatura, en relación con los contenidos apuntados en el mapa. Se va dando turno a todos los alumnos que deseen intervenir para sugerir esas ideas. Se dirige el debate en su parte final apuntando al futuro con optimismo, listando las principales soluciones acordadas, y motivando a los alumnos para superar la crisis periodística rescatando a la profesión, recuperando, fundamentalmente, la ética y deontología profesional y la contribución social del periodismo.

\title{
2.4.- Cuestionario de evaluación
}

En la clase anterior a la de comienzo de aplicación del CIMA, que en nuestro caso coincidía con la primera clase en este grupo (de presentación de nuestra parte de la asignatura), asi como también a la finalización de la última sesión, pedimos a los alumnos que, de forma anónima y virtualmente, completasen el siguiente cuestionario inicial de 5 preguntas abiertas:

Cuestionario de opiniones sobre aspectos relacionados con el periodismo social.

"Periodismo social: ¿para quién, para qué, por qué y cómo?"

Por favor, contesta el siguiente sondeo reflexionando con los conocimientos y opiniones que tengas actualmente, sin buscar información alguna, para que pueda ser de utilidad en el desarrollo de las próximas clases. Exprésate con total libertad y sinceridad. Se trata de un cuestionario anónimo, en el que se pide únicamente añadir un alias o pseudónimo inventado.

\begin{abstract}
Alias:
1.- ¿Qué prejuicios, estereotipos o ideas preconcebidas crees que existen en nuestro país en torno a los inmigrantes?

2.- ¿Qué relación o conexión crees que podría establecerse entre el periodismo y los numerosos delitos de odio que tienen lugar en España en la actualidad? Argumenta lo mejor posible tu respuesta.

3.- ¿A qué aspectos crees que puede deberse fundamentalmente la crisis de credibilidad del periodismo actualmente en España? Razónalo.

4.- ¿Cómo crees que podría el periodista hacer contribuciones sociales importantes al conjunto de la sociedad?. Trata de convencerme para que las haga.

5.- ¿Qué aspectos o elementos habría que observar en un texto periodístico para identificar si se ha dado un buen tratamiento informativo a un hecho o acontecimiento? Argumenta cada aspecto.
\end{abstract}

Figura 3. Cuestionario

Ciclos de Mejora en el Aula (2020). Experiencias de Innovación Docente de la US Esta obra se distribuye con la licencia Creative Commons Reconocimiento-NoComercial-SinObraDerivada Internacional (CC BY-NC-ND 4.0.) 


\section{Aplicación del CIMA}

\section{Desarrollo real de las sesiones}

De un modo general, la experiencia con la aplicación de este CIMA en las cuatro sesiones de clases ha sido muy positiva. Ha habido una alta asistencia, aunque esta haya ido progresivamente a menos $(59,57,55$ y 50 alumnos, respectivamente), una gran participación y un elevado grado de implicación, incluso más de lo previsto, sobre todo teniendo en cuenta las dificultades que plantea el sistema virtual de enseñanza y la poca familiaridad aún con el mismo por parte de todos. En definitiva, consideramos que han aprendido, que hemos desarrollado el modelo metodológico propuesto y que hemos cubierto el mapa de contenidos en su totalidad en estas cuatro sesiones, dándose respuesta a los principales problemas planteados en él y pudiendo desarrollarse las 10 actividades contempladas. Sin embargo, hemos echado en falta ese ambiente de calidez, cercanía y mayor agilidad que solo puede proporcionarnos el sistema presencial de enseñanza en un aula física, en el que pensamos que habríamos logrado con nuestra propuesta un mayor impacto y una más clara mejora, en relación con cursos anteriores.

Por otro lado, pese al cumplimiento general de las bases planteadas en el diseño de este CIMA, durante el desarrollo de las sesiones y de las actividades recogidas en la secuencia, surgieron algunos contratiempos que llevaron a realizar ligeras modificaciones, sobre la marcha, en el plan inicial previsto de actividades. En su inmensa mayoría, estos cambios fueron debidos en general a la falta de tiempo, por la mayor lentitud que implica realizar dinámicas y tareas en un sistema de enseñanza virtual, y algunos problemas técnicos de audio, de imagen o de conexión 
que se produjeron. Estos problemas provocaron pérdidas de tiempo en casi todas las actividades, repercutiendo en cadena en las posteriores y terminando las sesiones con cierta precipitación, lo que impidió hacer los debates o balances generales finales previstos, así como una mayor retroalimentación del resto de la clase, tras las distintas intervenciones o presentaciones de alumnos.

Así, por ejemplo, aunque la idea inicial era que los grupos de trabajo fueran cambiando para las actividades de las distintas sesiones, tuvimos finalmente que mantener los mismos grupos para las cuatro clases, compensando así parte del tiempo perdido, para poder cumplir con nuestro cronograma.

Además, hubo que recortar las duraciones inicialmente previstas de algunas actividades. En este sentido, el cambio más drástico se dio en la actividad 10, la última de la secuencia, reducida de 60 a 20 minutos por esa falta de tiempo. Así, en lugar de ir completando en esa sintesis final general una pizarra en blanco con una lluvia de ideas por parte de los alumnos, para el profesor ir añadiendo sobre ellas algunas nociones teóricas de la asignatura, se tuvo que pasar a proyectar el mapa de contenidos ya completo del diseño de las cuatro sesiones y, sobre él, el profesor fue poniendo en relación las distintas ideas y añadiendo nociones teóricas de la asignatura. Sin embargo, consideramos que fue efectivo este cambio y esta síntesis final, sobre todo dado el poco margen de tiempo con el que contábamos al final de la cuarta sesión y que era necesario poner en relación todas las ideas abordadas en las cuatro sesiones para dar coherencia, facilitar la comprensión y consolidar el aprendizaje.

Otro inconveniente surgido fue que hubo que atrasar la fecha de la sesión 4 por un examen que programaron en un horario que se solapaba con nuestra clase y ese excesivo tiempo transcurrido entre la tercera y la cuarta sesión creemos que afectó negativamente. Y es que este "enfriamiento" hizo que los alumnos olvidaran, de una semana para otra, algunas de sus notas y los ejemplos con los que 
habían trabajado en la actividad 7 y 8 (sesión 3), que tenían una clara continuidad en la actividad 9 (sesión 4).

Por otro lado, y como aspecto a mejorar, en la actividad 5 de resolución de problemas, los alumnos se centraron casi únicamente en los significados de los extractos, pero dejaron de lado u olvidaron debatir y analizar los efectos y las posibles soluciones, pese a que se les pidió por escrito y oralmente en la actividad. En este sentido, no hubo la deseable profundización en los análisis, aunque fueron interesantes las conclusiones a las que se llegó, en general, en relación con los significados.

Otro aspecto mejorable fue el hecho de que, pese a la alta participación, en los momentos en los que había que hablar en público a toda la clase, activando únicamente el micrófono, los protagonistas eran casi siempre los mismos pocos estudiantes. En este sentido, hubo cierta timidez, pidiendo muchos alumnos escribir preferiblemente por el chat de la sala sus valoraciones o intervenciones. En relación también con este aspecto, algunos grupos tardaron más tiempo de lo previsto en decidir quién sería el portavoz y/o no se pusieron de acuerdo para elegir a uno en algunas actividades.

Por último, con respecto a los cuestionarios anónimos realizados con anterioridad y posteridad a la aplicación de este CIMA, cambiaríamos el modo en el que los realizamos, ya que, pese a que el inicial fue completado y validado (no habiendo olvidado el sujeto asignar un alias y habiendo borrado las huellas de autoría que pudiesen identificarle) por hasta 45 alumnos (de los 71 matriculados, de los que asistieron con regularidad algo más de 50), el resultado final no ha sido muy positivo. Y es que de los cuestionarios finales anónimos recibidos (27 en total), solo pudieron validarse 18, por no haberse olvidado incluir el mismo alias que en el inicial, por haberse borrado las huellas de autoría y por haber realizado también ese mismo sujeto el

Ciclos de Mejora en el Aula (2020). Experiencias de Innovación Docente de la US Esta obra se distribuye con la licencia Creative Commons 
cuestionario inicial (para poder así estudiar su evolución en la escalera de aprendizaje).

En este sentido, creemos que a la escasa participación en esta evaluación, o a los problemas que han llevado a dar por válido un número de cuestionarios escaso (en comparación con el total de alumnos asistentes), ha podido contribuir el sistema empleado para su realización. Al no dar la plataforma de enseñanza virtual la opción de una entrega anónima por parte de los alumnos, estos tuvieron que descargar el cuestionario de la plataforma en formato .doc, completarlo, borrar las huellas de autoría del archivo y remitírselo por correo electrónico a alguno de los tres voluntarios que recopilarían todos los cuestionarios de los demás compañeros para enviárselo al profesor, asegurando así el anonimato de los mismos.

Y también ha podido contribuir a ello las circunstancias en las que se realizó el cuestionario final, pidiéndolo como tarea, ya fuera del horario de clase, por no haber dado tiempo en la última sesión del CIMA a realizarlo.

\section{2.- Evaluación del aprendizaje de los estudiantes}

En las Figuras 3 a 7 siguientes se pueden observar los resultados comparativos de los cuestionarios iniciales y finales de evaluación en forma de escaleras de aprendizaje, para cada una de las 5 preguntas de las que consta el mismo, con indicación de los distintos obstáculos a superar por los alumnos para subir de peldaños:

Ciclos de Mejora en el Aula (2020). Experiencias de Innovación Docente de la US Esta obra se distribuye con la licencia Creative Commons 
PREGUNTA 1.- ¿Qué prejuicios, estereotipos o ideas preconcebidas crees que existen en nuestro país en torno a los inmigrantes?

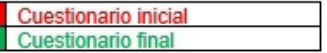

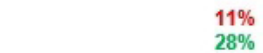

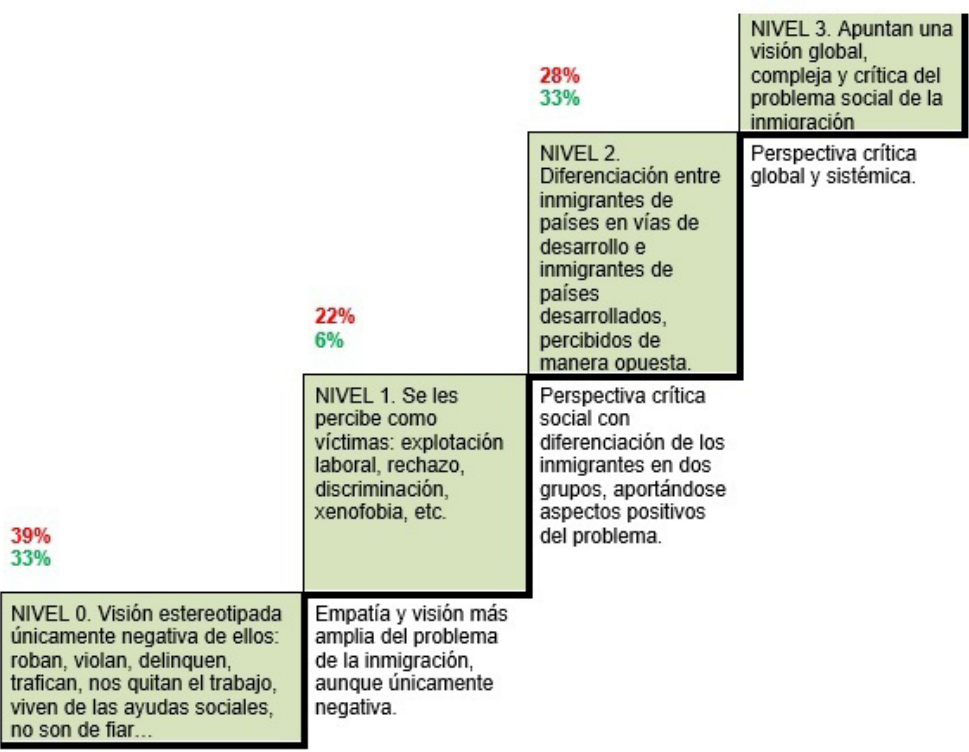

Figura 4. Resultados de la pregunta 1

PREGUNTA 2.- ¿Qué relación o conexión crees que podría establecerse entre el periodismo y los numerosos delitos de odio que tienen lugar en España en la actualidad? Argumenta lo mejor posible tu respuesta.

\begin{tabular}{|l|l|}
\hline Cuestionario inicial \\
\hline Cuestionario final \\
\hline
\end{tabular}

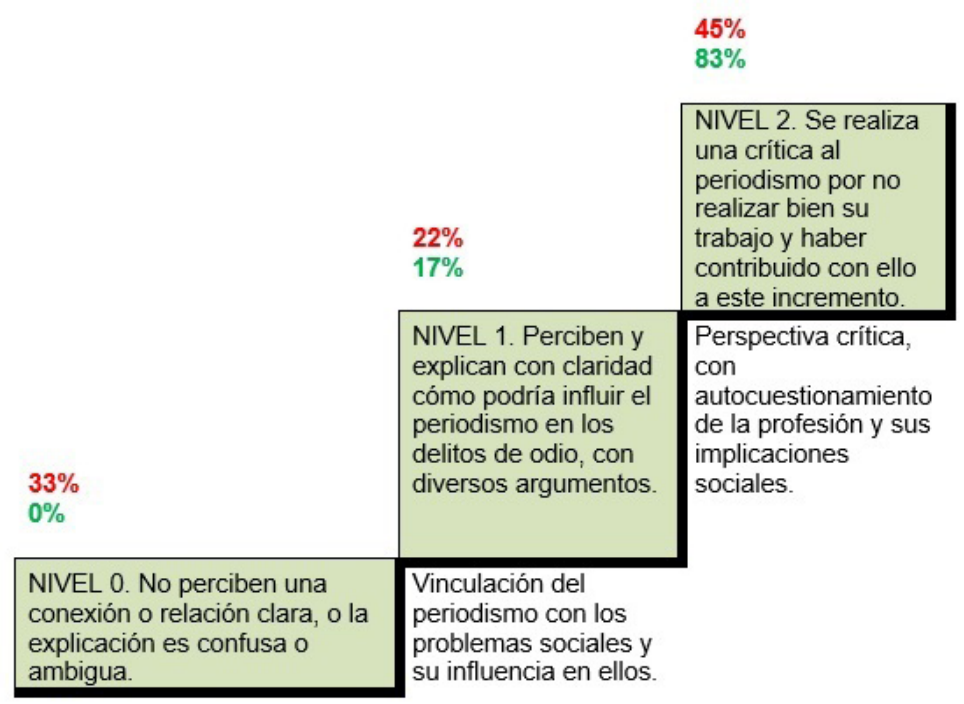

Figura 5. Escalera aprendizaje pregunta 2.jpg

Ciclos de Mejora en el Aula (2020). Experiencias de Innovación Docente de la US Esta obra se distribuye con la licencia Creative Commons Reconocimiento-NoComercial-SinObraDerivada Internacional (CC BY-NC-ND 4.0.) 
PREGUNTA 3.- ¿A qué aspectos crees que puede deberse fundamentalmente la crisis de credibilidad del periodismo actualmente en España? Razónalo.
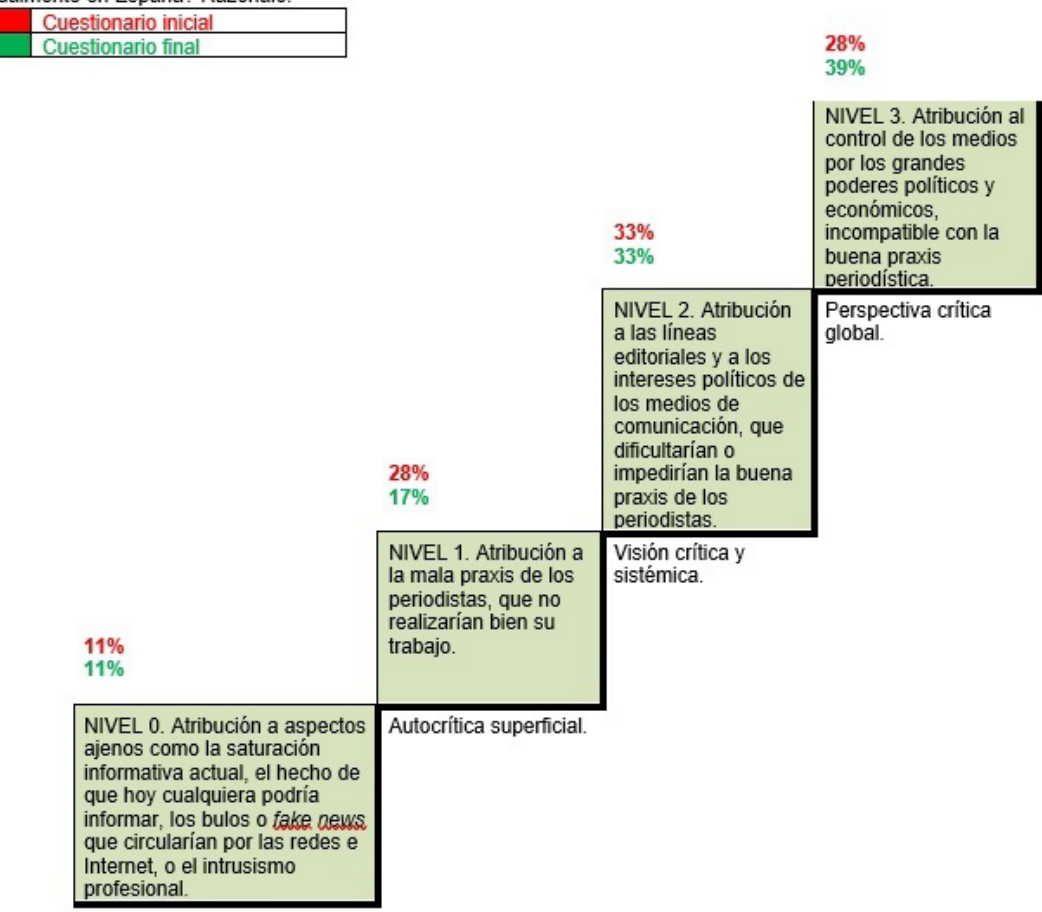

Figura 6. Resultados de la pregunta 3

PREGUNTA 4- ¿Cómo crees que podría el periodista hacer contribuciones sociales importantes al conjunto de la sociedad?. Trata de convencerme para que las haga.

\section{Cuestionario inicial}

$39 \%$

$50 \%$

NIVEL 3. No

sucumbiendo a las

presiones e intereses

de los poderes

económicos y

políticos que

controlarían los

medios, y siendo

críticos en las informaciones.

NIVEL 2. Realizando

Perspectiva crítica

global o sistémica y

buenos trabajos

periodísticos en

general, más allá de

lo social:

imparciales

profundos, rigurosos,

éticos, etc.

$0 \%$

NIVEL 1. Ayudando a

Autocrítica de la

la gente que lo

mala praxis

necesitaría y

denunciando

injusticias sociales,

que sería una misión

del periodismo

Empatía y visión de la

función social del

periodismo.

Figura 7. Resultados de la pregunta 4

Ciclos de Mejora en el Aula (2020). Experiencias de Innovación Docente de la US Esta obra se distribuye con la licencia Creative Commons Reconocimiento-NoComercial-SinObraDerivada Internacional (CC BY-NC-ND 4.0.) 
PREGUNTA 5- ¿Qué aspectos o elementos habría que observar en un texto periodístico para identificar si se ha dado un buen tratamiento informativo a un hecho o acontecimiento? Argumenta cada aspecto.

\section{Cuestionario inicial}

Cuestionario final

$0 \%$

$28 \%$

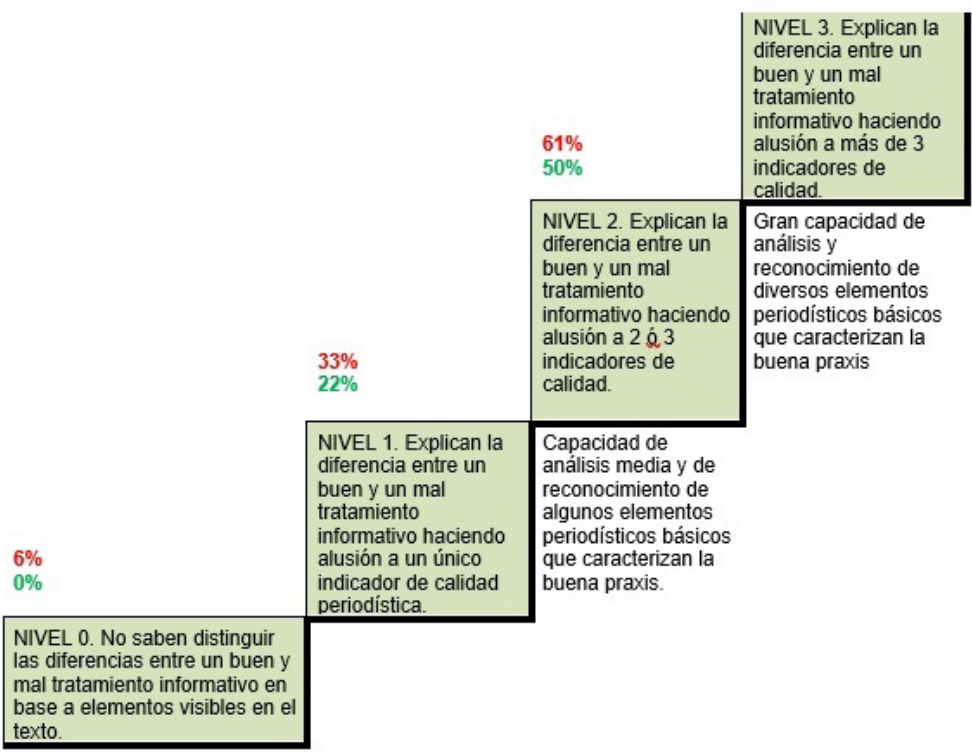

Figura 8. Resultados de la pregunta 5

A continuación, realizaremos una valoración de los resultados de los cuestionarios por preguntas y por sujeto-alumno, comentando cómo ha sido la evolución del aprendizaje de estos tras la aplicación del CIMA.

En la pregunta 1 del cuestionario, un 39\% de los alumnos han superado obstáculos y subido de peldaño en la escalera de aprendizaje (un 6\% hasta tres peldaños, un $17 \%$ hasta dos escalones y un $17 \%$ solo uno), el $50 \%$ se ha mantenido en el mismo escalón y no habría superado obstáculos, y el 11\% habría bajado de peldaño (un 6\% un escalón y un $6 \%$ hasta tres), ateniéndonos a los cuestionarios finales e iniciales entregados por los estudiantes.

En la pregunta 2, un 45\% de los sujetos han superado obstáculos y subido de peldaño (un 33\% hasta dos escalones y un $11 \%$ solo uno), el $50 \%$ se ha mantenido en el mismo escalón y no habrían superado obstáculos, y el 6\% habría bajado de peldaño un escalón.

Ciclos de Mejora en el Aula (2020). Experiencias de Innovación Docente de la US Esta obra se distribuye con la licencia Creative Commons 
En la pregunta 3, un $22 \%$ de los alumnos han superado obstáculos y subido de peldaño (un 6\% hasta dos escalones y un $17 \%$ solo uno), un $61 \%$ se han mantenido en el mismo escalón y no habrían superado obstáculos, y un 17\% habrían bajado un peldaño.

En la pregunta 4, un 33\% de los estudiantes han superado obstáculos y subido de peldaño (un 6\% hasta tres escalones, un $11 \%$ hasta dos y un $17 \%$ solo uno), un $56 \%$ se han mantenido en el mismo escalón y no habrían superado obstáculos, y un 11\% habrían bajado un peldaño.

En la pregunta 5, un $50 \%$ de los alumnos han superado obstáculos y subido de peldaño (un 6\% hasta cuatro escalones, un $6 \%$ hasta tres, un $6 \%$ dos y un $33 \%$ solo uno), un $44 \%$ se han mantenido en el mismo escalón y no habrían superado obstáculos, y un 6\% habría bajado un peldaño.

En definitiva, se observa, de modo general, una evolución positiva en el aprendizaje de los estudiantes. Así, en cuatro de las cinco preguntas un tercio o más de la clase habría evolucionado positivamente y superado nuevos obstáculos (en dos de ellas, la mitad o más de la clase). No obstante, es la pregunta 3 la que arrojaría peores resultados, al evolucionar positivamente en su aprendizaje solo algo más de un quinto de la clase. Cabe recordar que esta pregunta estaría directamente relacionada con la actividad número 5 de la secuencia del CIMA, sobre la que apuntamos un problema en el subepígrafe anterior que trataremos de corregir en próximos diseños.

\section{Evaluación del CIMA y principios didácticos personales}

En primer lugar, en cuanto a las fortalezas, destacamos el positivo cambio de dinámica y metodología en la asignatura, suscitando un gran interés y una alta participación e implicación por parte de los alumnos, en uno de sus temas iniciales e introductorios, en ocasiones anteriores más aburrido y menos dinámico, por centrarse quizás 
en exceso en la teoría, y en el que los estudiantes no asumían un rol tan activo y tan protagonista. Por ello, y por la evolución positiva en el aprendizaje de los alumnos, mantendríamos en un futuro CIMA el mismo modelo metodológico, idéntico mapa de contenidos y problemas y el diseño de todas las actividades, aunque retocando ligeramente algunos aspectos de estas.

Así, en cuanto a las debilidades, muy relacionadas en nuestra opinión con la falta de tiempo y cierta precipitación final en las sesiones, debidas a la lentitud, distanciamiento, frialdad y dificultades que supone el sistema de enseñanza virtual utilizado excepcionalmente en este curso académico, destacamos la ya apuntada escasa diversidad de la participación en los momentos de hablar en público a toda la clase (especialmente en las actividades 4, 6 y 9) y la poca profundidad en los análisis realizados en la actividad 5, motivada probablemente por no haber dedicado a ella quizás el tiempo suficiente y/o no haber podido supervisar lo debido el trabajo de los grupos. También apuntamos como debilidad la escasa retroalimentación de la clase tras las distintas presentaciones o exposiciones de los portavoces de los grupos, si bien es cierto que, en esta ocasión, esa falta de tiempo y cierta precipitación final no lo habrían permitido.

Por tanto, consideramos importante apuntar las siguientes mejoras para próximos CIMAs: pedir más profundización en los análisis y supervisar más, en ese sentido, el trabajo de cada grupo; modificar los grupos de trabajo en las distintas actividades (aunque trayéndolos ya establecidos antes de cada clase, para evitar pérdidas de tiempo); incentivar una mayor diversidad en la participación (alternando obligatoriamente quizás las portavocías de los grupos o llamando a los alumnos aleatoriamente); cambiar el sistema de realización y entrega de cuestionarios de evaluación inicial y final; e incluir una mayor componente visual en las clases, ya que la mayor parte discurría por el canal sonoro, pudiendo resultar en algunos momentos algo frío, monótono o insulso.

Ciclos de Mejora en el Aula (2020). Experiencias de Innovación Docente de la US Esta obra se distribuye con la licencia Creative Commons 
Por último, y a modo de síntesis, tras el diseño, aplicación y evaluación de este CIMA, podemos resumir nuestros principios didácticos personales (y el esqueleto, por tanto, de nuestro modelo didáctico actual), en el momento actual, en los siguientes: conocer los modelos mentales de los estudiantes como punto de partida esencial (Porlán, 2017); no dar todo hecho a los alumnos, sino que descubran ellos mismos, convirtiéndose en protagonistas de su propio aprendizaje (Vygotsky, 1978; Bruner, 1978); que estos se impliquen, esfuercen y participen activamente en las clases; crear en ellas, en términos de Bain (2005:114-115), un entorno para el "aprendizaje crítico natural"; prescindir del tradicional esquema de las clases magistrales para ir alternando una gran diversidad de métodos más dinámicos, con protagonismo del centrado en el denominado por Finkel (2008:168) como "taller conceptual" o la resolución de problemas, así como la exploración o investigación, el análisis crítico y el trabajo en equipo (de menor a mayor escala, progresivamente); fomentar una actitud y un pensamiento crítico y complejo; fomentar la ética y los valores (honestidad, tolerancia y respeto, igualdad, empatía y solidaridad); motivar a los alumnos y tratarles con calidez y cercanía.

Palabras clave: periodismo social, periodismo, docencia universitaria, experimentación docente universitaria, innovación docente.

Keywords: social journalism, journalism, university teaching, university teaching experimentation, teaching innovation.

Ciclos de Mejora en el Aula (2020). Experiencias de Innovación Docente de la US Esta obra se distribuye con la licencia Creative Commons 


\section{Referencias bibliográficas}

Asensio-Pérez, P. y Domínguez-Delgado, R. (2020). A social journalism? Trump's Immigration Order on Spanish press. Proceedings of the Association for Information Science and Technology, 57, e 406, 2020.

Bain, K. (2005). Lo que hacen los mejores profesores de universidad (Traducido por O. Barberá). Valencia: Universitat de Valencia.

Bruner, J. (1978). The role of dialogue in language acquisition. En A. Sinclair, R.J. Jarvelle, y W.J.M. Levelt (Eds.), The Child's Concept of Language. New York: Springer-Verlag.

Cruz Roja Española (2015). Informe sobre la vulnerabilidad social 2014. Madrid: Cruz Roja. Recuperado de http://www.cruzroja.es/principal/ documents/449219/451193/IVS+2014+vs+interactiva. pdf/71b3cd58-9cd5-43fe-a75e-c3ed4b0b5006

Castillo-Tamayo, S. y Domínguez-Delgado, R. (2020). El tratamiento informativo de la inmigración en el caso tarajal en El País y ABC. En J.C. Suárez-Villegas y S. Marín-Conejo, Ética, Comunicación y Género. Debates Actuales. Madrid: Dykinson (pp. 285-298).

Cytrynblum, A. (2009). Periodismo social: una nueva disciplina. Buenos Aires: La Crujía.

Federación de Asociaciones de Periodistas de España (FAPE) (2017). Código Deontológico. Recuperado de http://fape.es/home/codigo-deontologico/

Finkel, D. (2008). Dar clase con la boca cerrada (Traducción de O. Barberá). Barcelona: Publicacions de la Universitat de València.

Gómez Mompart, J.L.; Gutiérrez Lozano, J.F.; Palau Sampío, D. (Eds.) (2013). La calidad periodística: teorías, investigaciones y sugerencias profesionales. Castelló de la Plana: Publicacions de la Universitat Jaume I.

Labio, A. (2006). Del estereotipo al amarillismo. Prácticas periodísticas incorrectas en el tratamiento de grupos sociales vulnerables. En Anàlisi:_quaderns de comunicació i cultura, no 33, 31-44.

López-Hernández, M. y Domínguez-Delgado, R. (2012). El valor documental del periodista como autor. Estudios sobre el mensaje periodístico, 18(2), 791-803. Ciclos de Mejora en el Aula (2020). Experiencias de Innovación Docente de la US
Esta obra se distribuye con la licencia Creative Commons Reconocimiento-NoComercial-SinObraDerivada Internacional (CC BY-NC-ND 4.0.) 
Porlán, R. (Coord.) (2017). Enseñanza universitaria. Cómo mejorarla. Madrid: Morata.

Porlán, R. y Alba-Fernández, N.D. (2020). Docentes universitarios: una formación centrada en la práctica. Madrid: Morata.

Raigón Pérez De la Concha, G. (2007). Introducción al periodismo social y educativo. Sevilla: Universidad de Sevilla.

Red Acoge (2014). Inmigracionalismo. Hagamos autocrítica: medios de comunicación libres de xenofobia. Madrid: Red Acoge. Recuperado de

http://redacoge.org/mm/file/Informelnmigracionismo-RedAcoge.pdf

Rodríguez Rey, A. et al (2015). La calidad de los medios y el uso de fuentes periodísticas en la prensa local de referencia en España. Estudios Sobre el Mensaje Periodístico, 21, 85-100.

Serrano, P. (2010). Desinformación. Cómo los medios ocultan el mundo. Barcelona: Península.

Vygotsky, L.S. (1978). Mind in society: The development of higher psychological processes. Cambridge: Harvard University Press.

Ciclos de Mejora en el Aula (2020). Experiencias de Innovación Docente de la US Esta obra se distribuye con la licencia Creative Commons 